\title{
Inhibition of Serotonergic Neurons in the Nucleus Paragigantocellularis Lateralis Fragments Sleep and Decreases Rapid Eye Movement Sleep in the Piglet: Implications for Sudden Infant Death Syndrome
}

\author{
Robert A. Darnall, ${ }^{1}$ Michael B. Harris, ${ }^{2}$ W. Hugh Gill, ${ }^{3}$ Jill M. Hoffman, ${ }^{1}$ Justin W. Brown, ${ }^{1}$ and Mary M. Niblock ${ }^{1}$ \\ ${ }^{1}$ Department of Physiology, Dartmouth Medical School, Lebanon, New Hampshire 03756, ${ }^{2}$ Institute of Arctic Biology, University of Alaska Fairbanks, \\ Fairbanks, Alaska 99775-7000, and ${ }^{3}$ Tulane School of Medicine, New Orleans, Louisiana 70115
}

\begin{abstract}
Serotonergic receptor binding is altered in the medullary serotonergic nuclei, including the paragigantocellularis lateralis (PGCL), in many infants who die of sudden infant death syndrome (SIDS). The PGCL receives inputs from many sites in the caudal brainstem and projects to the spinal cord and to more rostral areas important for arousal and vigilance. We have shown previously that local unilateral nonspecific neuronal inhibition in this region with $\mathrm{GABA}_{\mathrm{A}}$ agonists disrupts sleep architecture. We hypothesized that specifically inhibiting serotonergic activity in the PGCL would result in less sleep and heightened vigilance. We analyzed sleep before and after unilaterally dialyzing the 5- $\mathrm{HT}_{1 \mathrm{~A}}$ agonist ( \pm )-8-hydroxy-2-(dipropylamino)-tetralin (8-OH-DPAT) into the juxtafacial PGCL in conscious newborn piglets. 8-OH-DPAT dialysis resulted in fragmented sleep with an increase in the number and a decrease in the duration of bouts of nonrapid eye movement (NREM) sleep and a marked decrease in amount of rapid eye movement (REM) sleep. After 8-OH-DPAT dialysis, there were decreases in body movements, including shivering, during NREM sleep; body temperature and heart rate also decreased. The effects of 8-OH-DPAT were blocked by local pretreatment with $N$-[2-[4-(2-methoxyphenyl)-1-piperazinyl]ethyl]- $N$-2pyridinylcyclohexane-carboxamide, a selective $5-\mathrm{HT}_{1 \mathrm{~A}}$ antagonist. Destruction of serotonergic neurons with 5,7-DHT resulted in fragmented sleep and eliminated the effects of subsequent 8-0H-DPAT dialysis on REM but not the effects on body temperature or heart rate. We conclude that neurons expressing 5- $\mathrm{HT}_{1 \mathrm{~A}}$ autoreceptors in the juxtafacial PGCL are involved in regulating or modulating sleep. Abnormalities in the function of these neurons may alter sleep homeostasis and contribute to the etiology of SIDS.
\end{abstract}

Key words: serotonin; sleep; sudden infant death syndrome; 5-HT ${ }_{1 \mathrm{~A}}$ receptor; REM; 8-OH-DPAT

\section{Introduction}

Sudden infant death syndrome (SIDS) remains the most common cause of death in postneonatal infancy. A major advance in our understanding of the etiology of SIDS has been the finding that many SIDS infants have decreased binding of muscarinic and kainate receptors in the arcuate nucleus at the ventral surface of the medulla (Kinney et al., 1995; Panigrahy et al., 1997) and abnormal binding to serotonergic (5-HT) receptors in the medullary serotonergic nuclei, including the paragigantocellularis lateralis (PGCL) (Panigrahy et al., 2000; Kinney et al., 2003). Dysfunction in these regions may increase the risk for SIDS by altering protective reflexes to stressors encountered during sleep such as hypercapnia, hypoxia, and laryngeal stimulation (Filiano and Kinney, 1994). In conscious animals, the local dialysis of

Received May 3, 2005; revised July 19, 2005; accepted July 23, 2005.

This work was supported by National Institutes of Health Grants P01 HD036379 and R01 HD045653 and First Candle/SIDS Alliance Grant SP0030. We thank David S. Paterson for double labeling of TPOH and 5-HT 1 A receptors, Hannah C. Kinney for her support and research guidance, and Laurie Hildebrandt for her expert technical help.

Correspondence should be addressed to Dr. Robert A. Darnall, Department of Physiology, Borwell Building, 1 Medical Center Drive, Lebanon, NH 03756. E-mail: robert.a.darnall@hitchcock.org.

DOI:10.1523/JNEUROSCI.1770-05.2005

Copyright $\odot 2005$ Society for Neuroscience $\quad$ 0270-6474/05/258322-11\$15.00/0
$\mathrm{GABA}_{\mathrm{A}}$ agonists into the PGCL decreases the ventilatory response to hypercapnia (Curran et al., 2001) and prolongs the laryngeal chemoreflex (Van der Velde et al., 2003).

Sleep represents a period of relative vulnerability, when many control systems function at lower levels. Although SIDS presumably occurs during sleep, there is little evidence to support a specific sleep abnormality. Sleep research in SIDS has been focused on arousal as an important protective mechanism (Kahn et al., 2002). However, an increase in arousability may also be disadvantageous and lead to fragmented sleep or sleep deprivation (Simpson, 2001). We have reported previously that nonspecific neuronal inhibition with $\mathrm{GABA}_{\mathrm{A}}$ agonists in the PGCL region in the piglet disrupts sleep architecture and results in more wakefulness (WAKE) (Darnall et al., 2001).

The results of our previous experiments and the findings of altered serotonergic receptor binding in the PGCL in SIDS infants prompted us to look more closely at the role of PGCL 5-HT neurons in sleep. The PGCL extends caudally from the superior olive to the anterior pole of the lateral reticular nucleus (Andrezik et al., 1981). It receives inputs from the nucleus of the solitary tract, A1 region, parabrachial nucleus, Kölliker-Fuse nucleus, periaqueductal gray, and the hypothalamus. In addition, the 
more rostral (juxtafacial) PGCL receives polymodal sensory inputs from the inferior colliculus, the dorsal column nuclei, and the medial geniculate nucleus (Van Bockstaele et al., 1989, 1993; Van Bockstaele and Aston-Jones, 1995). The PGCL sends important projections to more rostral areas important for alertness and arousal, including the locus ceruleus (LC) (Aston-Jones et al., 1986, 1991a), and to both the dorsal and ventral horns of the spinal cord (Holstege and Kuypers, 1987) with extensive collateralization supplying multiple spinal cord segments (Bowker and Abbott, 1990; Kausz, 1991). Thus, neurons located in the PGCL are positioned to play an important role in integrating multiple sensory inputs for modulating brain and spinal alerting systems. In this study, we focused on 5-HT neurons in the juxtafacial PGCL and asked whether decreasing serotonergic activity would alter normal sleep patterns. Our paradigm was to evaluate sleep after unilaterally decreasing serotonergic activity in the PGCL using the selective $5-\mathrm{HT}_{1 \mathrm{~A}}$ agonist ( \pm )-8-hydroxy-2(dipropylamino)-tetralin (8-OH-DPAT).

\section{Materials and Methods}

Experiments were performed on piglets of either sex, aged 4-18 d and weighing $1.5-3.8 \mathrm{~kg}$ at the time of study. All surgery and experimental protocols were approved by The Institutional Animal Care and Use Committee of Dartmouth College. When not in the laboratory, the piglets were housed with the sow and siblings in a farrowing crate located in the Dartmouth College Animal Resource Center and were provided with a constant temperature and $12 \mathrm{~h}$ light/dark cycle. Piglets were brought to the laboratory on 1 or more days before surgery to acclimatize them to the experimental environment.

Surgical instrumentation. Our surgical procedures have been described in detail previously (Curran et al., 2001; Darnall et al., 2001). Briefly, under sterile conditions and using isofluorane anesthesia, a dual-lumen catheter was placed through the femoral artery into the abdominal aorta, and a telemetric thermistor was placed subcutaneously just lateral to the abdominal midline. After placement in a stereotaxic apparatus, a microdialysis guide tube was placed through a burr hole in the skull using coordinates derived from a regression formula (Sun et al., 2000). An attempt was made to place the tip between the midline and the medial border of the facial nucleus near the ventral surface. EEG electrodes were screwed into the left frontal and right occipital regions of the skull and referenced to a right parietal electrode. Electro-oculogram (EOG) electrodes were sewn in place lateral to and one above and one below each eye. Bipolar EMG electrodes were sewn deep into the neck muscles. All wires from the electrodes were attached to brass contacts inserted into two plastic pedestals, fixed to the skull with the microdialysis guide tube with cement. The femoral catheter was tunneled through the skin and exited on the back. After surgery, the animals were provided with analgesia and antibiotics, allowed to recover, and returned to the sow and siblings in the animal care facility.

Measurements. The animals were first studied $24-48 \mathrm{~h}$ after surgery. The piglet was suspended in a sling inside a barometric plethysmograph (Drorbaugh and Fenn, 1955; Bartlett and Tenney, 1970) modified to allow continuous gas flow (Pappenheimer, 1977). Air flowing through the plethysmograph was heated $\left(\sim 38^{\circ} \mathrm{C}\right)$ and fully humidified. The plethysmograph wall temperature was adjusted according to the weight and age of the animal using a circulating water bath to provide a thermoneutral environment. Heart rate (HR) and mean arterial pressure (BPm) were calculated from continuous measurements of arterial pressure (World Precision Instruments, Sarasota, FL). Respiratory measurements were derived from plethysmograph pressure fluctuations (Validyne, Northridge, CA). EEG, EOG, and EMG signals were amplified and bandpass filtered $(0.1-300 \mathrm{~Hz}$ for EEG and EOG and $10-300 \mathrm{~Hz}$ for EMG). The fractional $\mathrm{O}_{2}$ content of inlet and outlet air (model S-3A/II; Applied Electrochemistry, Pittsburg, $\mathrm{PA}$ ) and the fractional content of $\mathrm{CO}_{2}$ in the outlet air (Capstar-100; CWI, Ardmore, PA) were measured continuously to calculate oxygen consumption $\left(\mathrm{VO}_{2}\right)$ and carbon dioxide production $\left(\mathrm{VCO}_{2}\right)$. Plethysmograph air and animal core temperatures were continuously measured (YSI, Yellow Springs, OH and DSI, St. Paul, $\mathrm{MN}$ ). All signals were digitized at $1000 \mathrm{~Hz}$ and recorded using a computerized data acquisition system (PowerLab; ADInstruments, Castle Hill, Australia). Throughout the experiment, piglet behavior was video recorded and digitized for later sleep scoring.

Protocols. Animals were studied for 1-10 d after surgery. Based on previous observations showing that young newborn piglets sleep in bouts throughout the $24 \mathrm{~h}$ period with little consolidation during the dark cycle, all studies were performed between 10:00 A.M. and 3:00 P.M. and were of similar recording duration. The plethysmograph was sealed $\sim 1.5$ $\mathrm{h}$ before the experiment to allow the temperature and humidity to stabilize. Calibration was performed using sequential triplicate injections of 1 , 2,3 , and $5 \mathrm{ml}$ of air. The piglet was then placed in the plethysmograph and connected to the monitoring equipment. The microdialysis probe was inserted and dialysis started with artificial CSF (aCSF) [containing the following (in $\mathrm{mm}$ ): $152.2 \mathrm{Na}, 3.0 \mathrm{~K}, 131.1 \mathrm{Cl}$, and $1.5 \mathrm{Ca}$, adjusted to a $\mathrm{pH}$ of 7.4 ] at a flow rate of $8.5 \mu \mathrm{l} / \mathrm{min}$. After temperature, humidity, $\left[\mathrm{CO}_{2}\right]$, and $\left[\mathrm{O}_{2}\right]$ reached stable values $(\sim 1 \mathrm{~h})$, measurements were begun. Two protocols were used in this study: (1) an experimental protocol in which normal sleep cycling was first recorded for $\sim 2 \mathrm{~h}$, during which aCSF was continuously dialyzed. The dialysate was then switched to either 10 or 30 mm 8-OH-DPAT (Sigma, St. Louis, MO) and continued for $30 \mathrm{~min}$. The dialysate was then switched back to aCSF for the remainder of the experiment; and (2) a time-control protocol in which aCSF was substituted for the period of 8-OH-DPAT dialysis resulting in continuous aCSF dialysis throughout the experiment.

To confirm that our results were secondary to activating $5-\mathrm{HT}_{1 \mathrm{~A}}$ receptors, four animals were dialyzed for $30 \mathrm{~min}$ with $30 \mathrm{~mm}$ 8-OH-DPAT after pretreatment for $30 \mathrm{~min}$ of local dialysis with $\mathrm{N}$-[2-[4-(2methoxyphenyl)-1-piperazinyl] ethyl]-N-2-pyridinylcyclohexanecarboxamide (WAY100635; Sigma), a selective, "silent" $5-\mathrm{HT}_{1 \mathrm{~A}}$ receptor antagonist. The term silent $5-\mathrm{HT}_{1 \mathrm{~A}}$-receptor antagonist has been used to distinguish true antagonists from partial agonists and describes compounds that lack intrinsic activity yet effectively block the effects of receptor agonists. To further determine whether our results were attributable to activating somato-dendritic $5-\mathrm{HT}_{1 \mathrm{~A}}$ autoreceptors or postsynaptic 5- $\mathrm{HT}_{1 \mathrm{~A}}$ receptors, 5,7-dihydroxytryptamine (5,7-DHT; Sigma), a toxin selective for 5-HT neurons, was dialyzed into the PGCL of four animals after pretreatment with desipramine hydrochloride to prevent destruction of catecholaminergic neurons. After a week, $30 \mathrm{~mm}$ 8-OH-DPAT was dialyzed into the same region of the PGCL, and the results were compared with a group of animals of similar ages not treated with 5,7-DHT.

8-OH-DPAT doses. Relatively large doses of 8-OH-DPAT were used in the current study compared with those used in other dialysis experiments in the dorsal raphé (Portas et al., 1996). Keeping in mind that the estimated tissue concentration is $\sim 1 / 10$ of the dialysate concentration (De Lange et al., 1995), $10 \mathrm{~mm}$ 8-OH-DPAT (estimated 1 mм tissue concentration) produced some effects on sleep, but $30 \mathrm{~mm}$ was necessary to obtain consistent results. These doses are consistent with those used by other investigators in the caudal brainstem (Berner et al., 1999), and larger doses may be necessary for a number of reasons. Compared with dorsal raphé 5-HT neurons, caudal medullary 5-HT neurons have faster firing rates (Heym et al., 1982b), may have fewer 5- $\mathrm{HT}_{1 \mathrm{~A}}$ autoreceptors (Trulson and Frederickson, 1987), and appear to be less sensitive to 5-HT ${ }_{1 \mathrm{~A}}$ agonists (Heym et al., 1982a). Our fluorescein data and 5,7-DHT data, although not conclusive, provide some level of confidence that we were affecting $5-\mathrm{HT}_{1 \mathrm{~A}}$ receptors in an area restricted to the juxtafacial PGCL and a portion of the retrotrapezoid nucleus.

Data reduction and calculations. Data reduction, including sleep scoring, was done using custom programs written in Matlab (MathWorks, Natick, MA). For sleep-state scoring, a wavelet-based analysis that has been described previously (Darnall et al., 2001) was used to derive frequency information from the EEG. Slow wave $(\delta)$ activity was estimated by combining levels $6-9$ of a 9 level discrete wavelet decomposition/ reconstruction $(0.3-4.7 \mathrm{~Hz})$, and theta activity was estimated by using level $5(4.7-9.4 \mathrm{~Hz})$. A similar analysis was done for EOG and nuchal EMG recordings to isolate the most important features of each signal. Periods of non-rapid eye movement (NREM) sleep, rapid eye movement 
(REM) sleep, and WAKE were identified using the combination of EEG, EOG, nuchal EMG data, and behavioral criteria. An automated sleepscoring algorithm using criteria related to the changes in the delta and theta activity, presence of rapid eye movements, and nuchal EMG activity was used to identify areas most likely to be NREM, REM, or WAKE. REM sleep was identified as periods of low delta activity, an elevated ratio of theta/delta activity (TDratio), absent nuchal EMG activity, and the presence of rapid eye movements. NREM sleep was identified by evidence of increased delta activity, a low TDratio, intermediate nuchal EMG activity, and no rapid eye movements. The transition to WAKE was identified by a rapid increase in nuchal EMG activity and intermediate values for delta and the TDratio. An interactive program incorporating the digital video was then used to manually correct sleep-state transitions. Using this technique, $\sim 5 \%$ of the recording was left unscored.

For cardiorespiratory variables, the original digitized data were resampled at rates appropriate for the variable. For respiratory calculations, the maximum and minimum of each breath related pressure fluctuation were determined using an automated peak detector followed by manual correction, if necessary. The amplitude of each breath (maximum minimum) was used to derive breath to breath tidal volume $\left(V_{\mathrm{T}}\right)$ (Bartlett and Tenney, 1970). Minute ventilation $\left(V_{\mathrm{E}}\right)$ was calculated as the product of $V_{\mathrm{T}}$ and instantaneous respiratory rate (RR) calculated from the interbreath interval. The peak of each blood pressure pulse was determined similarly and was used to calculate beat-to-beat HR. BPm was calculated from the arterial pressure waveform.

For metabolic variables, body and plethysmograph temperature, inlet and outlet $\left[\mathrm{O}_{2}\right]$ and $\left[\mathrm{CO}_{2}\right]$, and plethysmograph gas flow rate were averaged in $1 \mathrm{~s}$ bins and smoothed using a $60 \mathrm{~s}$ time constant. $\mathrm{VO}_{2}$ was calculated from the difference in the fractional inlet and outlet $\left[\mathrm{O}_{2}\right]$ and the gas flow rate. $\mathrm{VCO}_{2}$ was similarly calculated assuming an inlet room air fractional concentration of 0.0003 . We made no attempt to correct the measurements for water content or the respiratory quotient (RQ) and assumed no major changes in either over the course of the experiments.

Neuroanatomy. At the conclusion of experiments, each piglet was killed with an injection of sodium pentobarbital followed by an intracardiac injection of $5-10 \mathrm{ml}$ of saturated potassium chloride. Microinjections of $20-50 \mu \mathrm{l}$ of $1 \%$ potassium permanganate were made through a broken microdialysis probe to mark the location of the tip of the microdialysis probe in reference to external landmarks (Sun et al., 2000). The brainstem was removed and frozen in cryoembedding medium (Tissue-Tek OCT; Sakura Finetek, Torrance, CA). Brainstems were cryosectioned $(40 \mu \mathrm{m})$ at $-18^{\circ} \mathrm{C}$, and sections were thaw-mounted on gelatin-coated glass slides. Sections were fixed for $10 \mathrm{~min}$ in $37 \%$ phosphate-buffered formalin, $\mathrm{pH} 7.4$, and then stained with cresyl violet.

The rostrocaudal length of the brainstem differed among piglets over the ages we studied, and coordinates expressed relative to the bregma or interaural line did not always accurately describe the location of dialysis probes with respect to internal medullary landmarks. Therefore, we expressed the location of each probe with respect to three relevant internal medullary structures: the midline, the ventral surface, and the caudal pole of the facial nucleus (Curran et al., 2001). For convenience, the lesions are plotted both in reference to the obex and the facial nucleus in the results section.

To determine the extent of the neuronal destruction caused by local dialysis of 5,7-DHT, immunohistochemical methods were used to identify neurons containing tryptophan hydroxylase (TPOH). TPOH immunohistochemistry in the piglet brainstem has been described in detail previously (Niblock et al., 2004). Adjacent sections were stained with cresyl violet for anatomical comparisons and identification of medullary nuclei and landmarks. In addition, neurons containing tyrosine hydroxylase $(\mathrm{TyrOH})$ were also identified using an identical protocol, with the exception that monoclonal mouse anti-rat tyrosine hydroxylase antibody (Sigma) was used as the primary antibody.

To determine the extent of the destruction of 5-HT neurons in the PGCL, TPOH-immunoreactive (TPOH-ir) cells were counted on the lesioned side within a standardized counting area and compared with counts within an identical counting area on the unlesioned side. Rectangles of equal dimensions were superimposed onto each slice that was used for counting, on either side of the midline. The rectangle extended later- ally from 1 to $4.2 \mathrm{~mm}$ lateral to the midline and from the ventral surface to $3.1 \mathrm{~mm}$ dorsal to the surface at the most medial dimension. Every sixth $40 \mu \mathrm{m}$ section was counted, extending from 18 sections caudal to the end of the lesion to 18 sections rostral to the most rostral edge of the lesion. In a few instances, there were areas of tissue destruction within the counting area caused by the dialysis guide tube. To correct for this, the mirror image of the damaged area was superimposed on the unlesioned side, and cells within this area were excluded from the cell count. Cells were counted only if they were within the counting area, morphologically identifiable as neurons, axon and dendrite(s) were visible, and the neuronal cytoplasm had a dense distribution of reaction product that excluded the nucleus if visible.

To determine whether 5 - $\mathrm{HT}_{1 \mathrm{~A}}$ receptors localize on 5-HT neurons in the PGCL, neurons were double labeled with antibodies for TPOH and the $5-\mathrm{HT}_{1 \mathrm{~A}}$ receptor. Forty micrometer sections were fixed in $4 \%$ paraformaldehyde at $4^{\circ} \mathrm{C}$ for $30 \mathrm{~min}$, blocked in $4 \%$ normal goat serum, and incubated in primary antibody overnight at $4^{\circ} \mathrm{C}$. Sections were washed in PBS with $0.1 \%$ Triton-X and incubated in appropriate anti-rabbit and anti-mouse fluorescent secondary antibodies (Alexa Fluor) for $2 \mathrm{~h}$ at room temperature. Sections were then washed in PBS with $0.1 \%$ Triton-X and allowed to dry for $60 \mathrm{~min}$ at room temperature before being coverslipped in Fluoromount-G (Southern Biotechnology, Birmingham, AL).

Analysis and statistics. Twenty-one animals of either sex were studied on different days to determine the effects of 8-OH-DPAT on sleep. Time control experiments were performed on six animals, in which aCSF was dialyzed for the entire study period. Six experiments were performed with $10 \mathrm{~mm} 8$ 8-OH-DPAT, and 17 experiments were performed using 30 mM 8-OH-DPAT. An additional four animals were studied with both WAY 100635 and 8-OH-DPAT, and the results were compared with both the six control and $1730 \mathrm{~mm}$ 8-OH-DPAT experiments. Another four animals were studied with $30 \mathrm{~mm}$ 8-OH-DPAT 1 week after dialysis of 5,7-DHT. The results of these experiments were compared with a subgroup of piglets from the $30 \mathrm{~mm}$ 8-OH-DPAT experiments that were $\geq 12 \mathrm{~d}$ of age at the time they were studied.

Probe tips were considered to in the appropriate position if there was a high likelihood that dialyzed 8-OH-DPAT would extend into the PGCL. We therefore accepted probe tip locations that were at least $1 \mathrm{~mm}$ lateral to the midline and $\leq 1.5 \mathrm{~mm}$ lateral to the medial edge of the facial nucleus. For the analysis, we treated each study day as a separate case. Bout number, bout duration, and the percentage of time spent in each state was determined for REM, NREM, and WAKE, as well as slow wave or delta activity, integrated EEG amplitude, and integrated neck EMG activity. Data were averaged for the hour before and the hour starting with the onset of 8-OH-DPAT (or sham aCSF) dialysis and entered into a three-way ANOVA (Systat version 10.2; Systat, Evanston, IL). The dialysate (aCSF or 8-OH-DPAT) and the state (NREM, REM, WAKE) were considered repeated or within-subjects factors, and the 8-OH-DPAT dose $(0,10$, or $30 \mathrm{~mm})$ was used as a grouping factor. In some analyses, age or a combination of age and weight was added as covariates in the analysis. Post hoc tests were performed if there were significant interactions between factors. Pairwise comparisons were done by computing a critical $T$ value from the MS error term (Winer, 1962) and correcting the resulting probability for multiple comparisons. Values were expressed as means \pm SEM, and the criterion for statistical significance was set at $p<$ 0.05 .

\section{Results}

\section{Distribution of 5-HT neurons in the piglet medulla and anatomic locations of dialysis probe tips}

The distribution of TPOH-ir neurons in the piglet brainstem and its relationship to other species, including the human, has been described in detail previously (Niblock et al., 2004). In the piglet, the PGCL contains 5-HT neurons that lie in a column lateral to the midline that extends from the ponto-medullary border to several millimeters caudal to the caudal pole of the facial nucleus. The more rostral (juxtafacial) portion of the PGCL lies mostly medial to the facial nucleus. In the dorsoventral dimension, it 
A
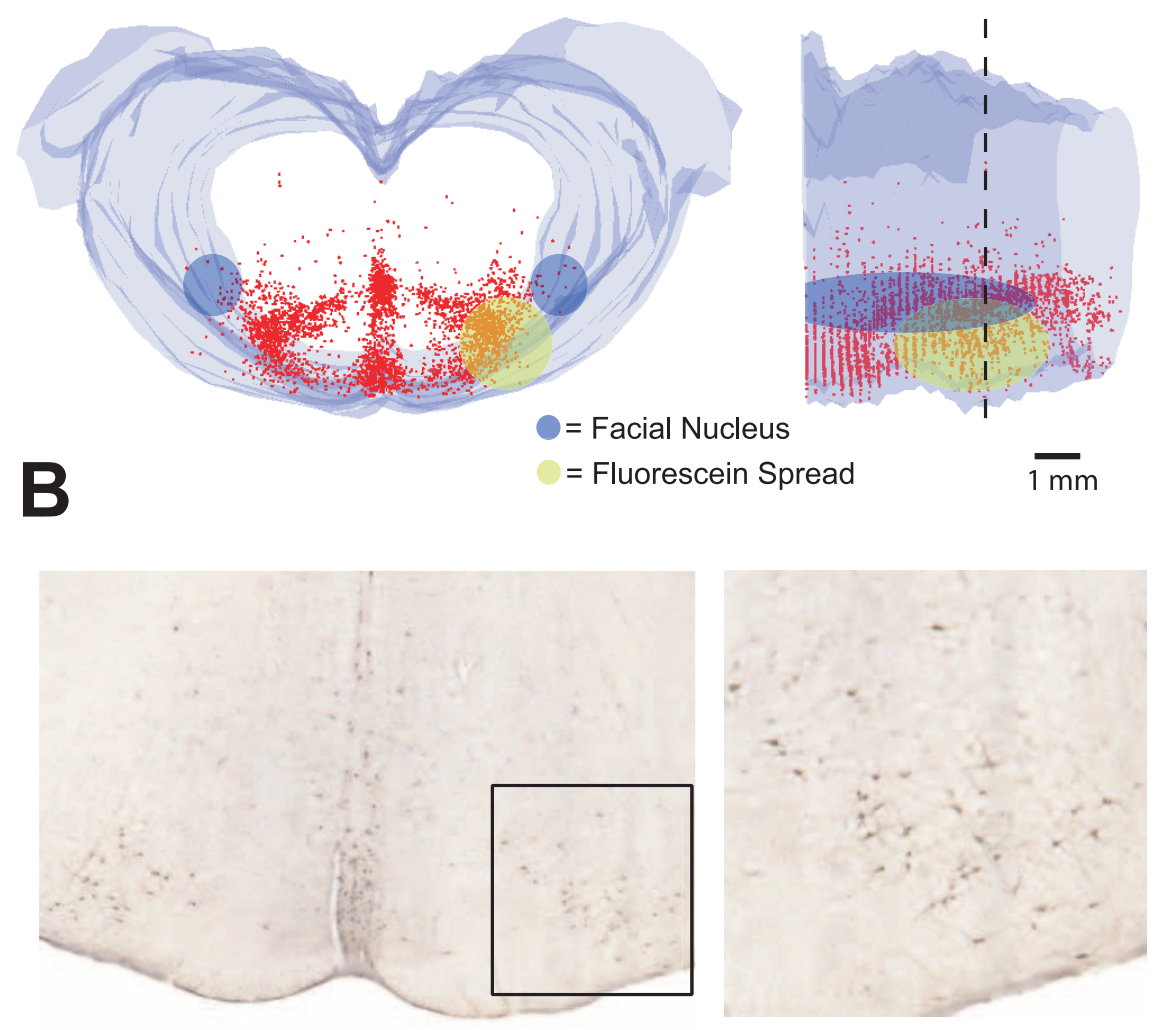

Figure 1. 3-D reconstruction of the piglet brainstem showing the distribution of 5-HT neurons. $A, A$ cross-sectional view is on the left, and a longitudinal view is on the right. TPOH-ir neurons are shown as red dots. The blue shaded areas are the approximate locations of the facial nuclei. The yellow-green area is the approximate diffusion area of dialyzed $30 \mathrm{~mm}$ 8-OH-DPAT centered in the juxtafacial PGCL as determined by fluorescein. $\boldsymbol{B}$, The left panel shows a section through the medulla near the caudal end of the facial nucleus (indicated by the dashed line in $\boldsymbol{A}$ ). TPOH-ir neurons are stained brown. The rectangle contains 5 - $\mathrm{HT}$ neurons located in the PGCL, which in this section lie lateral to the pyramids and medial to the facial nucleus. The right panel is an enlarged view of the area within the rectangle shown in the left panel.
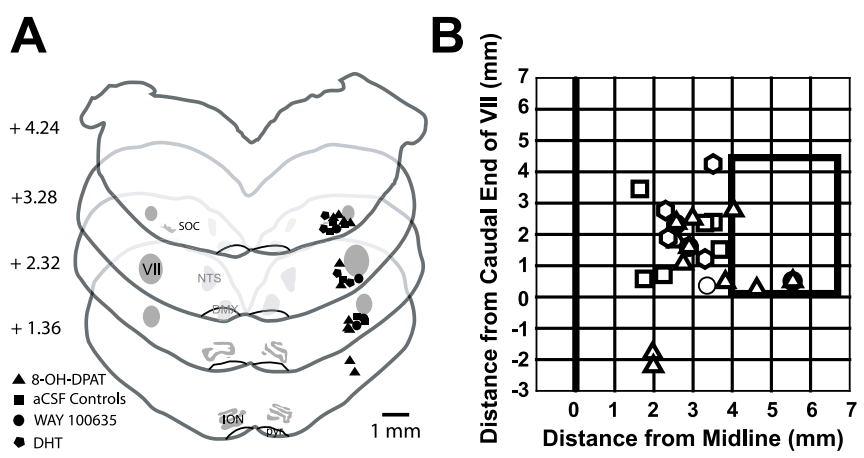

Figure 2. Location of the dialysis probe tips. $\boldsymbol{A}$, Location of the dialysis probe tips referenced to the obex. $\boldsymbol{B}$, Location of the dialysis probe tips referenced to a standardized facial nucleus to take growth and different sizes of the animals into account. The black rectangle represents the standardized facial nucleus. Probe tips located within the rostrocaudal dimension of the nucleus are plotted as absolute distances from the midline and relative distances between the caudal and rostral pole of the facial nucleus. SOC, Superior olivary complex; NTS, nucleus of the solitary tract; ION, inferior olive; pyr, pyramid; DHT, 5,7-DHT; VII, facial nucleus.

extends from the midline to near the ventral surface. Figure $1 \mathrm{~A}$ shows a three-dimensional (3-D) reconstruction of the piglet medulla showing the locations of TPOH-ir neurons (shown as red dots) in relation to the facial nucleus (shown in blue) and the approximate location and size of the distribution of dialyzed
8-OH-DPAT as determined by dialysis of $30 \mathrm{~mm}$ fluorescein (shown in yellow). The rationale for and problems associated with using fluorescein to determine distribution volume has been described previously (Curran et al., 2001). Nevertheless, the distribution of fluorescein provides a rough estimate of the extent of spread of dialyzed compounds of similar molecular weights. A 30 min dialysis of $30 \mathrm{~mm}$ fluorescein produced a $3-\mathrm{D}$ ellipse that was $\sim 2.1 \mathrm{~mm}$ wide and $\sim 3.5 \mathrm{~mm}$ long with a volume of $13.2 \mu \mathrm{l}$. This value is approximately twice as large as the volumes obtained in our laboratory using $10 \mathrm{~mm}$ fluorescein dialyzed for $20 \mathrm{~min}$ in chronically instrumented piglets $(5.8 \mu \mathrm{l})$ (Messier et al., 2002) or $10 \mathrm{~min}$ in decerebrate piglets (6.3 $\mu \mathrm{l})$ (Curran et al., 2000). A typical section at the level of the facial nucleus is shown in Figure $1 B$, illustrating the location of TPOH-ir neurons.

Figure $2 A$ shows the location of the dialysis probe tips in the study animals referenced to the obex, and Figure $2 B$ shows the rostrocaudal and mediolateral position of the probe tips in relation to a standardized facial nucleus as discussed in $\mathrm{Ma}$ terials and Methods. Note that most of the tip locations are clustered medial to the facial nucleus.

\section{Effect of 8-OH-DPAT on sleep architecture}

Our initial analysis using $0 \mathrm{~mm}$ (time control; aCSF), $10 \mathrm{~mm}$, and $30 \mathrm{~mm} 8-\mathrm{OH}-$ DPAT revealed significant interactions between dose, state, age, and the effect of 8-OH-DPAT on several measures of sleep architecture, including bout number, bout duration, and time spent in each state. However, the effect of $10 \mathrm{~mm} 8$-OH-DPAT was not consistently different from that of aCSF alone. In contrast, the effects of $30 \mathrm{~mm}$ 8-OH-DPAT on all variables were consistently different from both the effects of aCSF and $10 \mathrm{~mm} 8-\mathrm{OH}$-DPAT. We therefore elected to report in detail only the effects produced with $30 \mathrm{~mm}$ 8-OH-DPAT dialysis. Six animals were in the control group and received continuous dialysis with aCSF. They weighed $2.50 \pm$ $0.27 \mathrm{~kg}$ and were $8.3 \pm 1.5 \mathrm{~d}$ of age. In 17 experiments, animals were dialyzed with aCSF for $\sim 2 \mathrm{~h}$ and then were dialyzed with 30 mM 8-OH-DPAT for $30 \mathrm{~min}$ followed by aCSF dialysis for the remainder of the experiment. The animals in this group weighed $2.61 \pm 0.12 \mathrm{~kg}$ and were $10.3 \pm 0.8 \mathrm{~d}$ of age. There were no significant differences in the weights and ages of the two groups.

Because our studies were performed over an age range of 4-18 $\mathrm{d}$, we first examined the relationship between age and measures of sleep architecture. Over the age range of the piglets that we studied, there was no relationship between age and bout number, bout duration, or the percentage of time spent in any state. However, the effect of 8-OH-DPAT on the bout numbers of NREM $\left(R^{2}=0.353 ; p=0.012\right)$ and WAKE $\left(R^{2}=0.349 ; p=0.013\right)$, but not REM, was progressively smaller with increasing age. In contrast, there was no relationship between age and the effect of $8-\mathrm{OH}-\mathrm{DPAT}$ on bout duration or percentage of time spent in any 
state. Age-related effects were taken into account in subsequent analyses when appropriate.

The main effect of dialyzing 8-OHDPAT into the PGCL was fragmentation of sleep associated with a dramatic reduction in the amount of REM sleep. We defined sleep fragmentation as an increase in the number and a decrease in the duration of bouts of NREM sleep. Figure 3 shows data from a typical experiment. Note the regular cycling of sleep states before dialysis compared with the fragmented pattern during and shortly after 8-OH-DPAT dialysis. The most striking result was the marked decrease in the number of REM bouts and the percentage of time spent in REM after 8-OH-DPAT dialysis. REM was completely abolished in 53\% (9 of 17) of the piglets. In the remaining eight piglets, there was a $75.4 \%$ decrease in the number of REM bouts. Figure 4 shows the effects of 8-OH-DPAT dialysis on bout number, bout duration, and the percentage of time spent in each state. Note the decrease in REM bouts associated with the increase in number of both NREM and WAKE bouts. In addition, bouts of NREM were shorter, resulting in no difference in the percentage of time spent in NREM. In contrast, bouts of WAKE were more frequent, but not of shorter duration, resulting in a significant increase in the percentage of time spent in WAKE.

In addition, the shorter periods of NREM were associated with lower levels of delta activity and integrated EEG amplitude after 8-OH-DPAT dialysis compared with continuous aCSF dialysis experiments. In addition, most animals exhibited a decrease in motor activity and a decrease in muscle tone during NREM after 8-OH-DPAT dialysis. Although an attempt was made to provide a thermoneutral environment, some animals exhibited shivering during NREM and WAKE during control periods, indicating that they were below their thermoneutral range. In these cases, there was a marked decrease or absence of shivering after 8-OH-DPAT dialysis. Neck EMG activity, on average, was also lower after 8-OH-DPAT dialysis. However, the small number of subjects accompanied by the large variation in the measurement of nEMG precluded a meaningful comparison between the changes in nEMG in the control (sham aCSF) group and the 8-OH-DPAT group. Mean and individual data are shown in Figure 5 . Because of the possibility that periods that we scored as NREM after 8-OH-DPAT were examples of a dissociated state with REM-like hypotonia without rapid eye movements, we compared levels of neck EMG and EEG amplitude and $\delta$ activity during REM before 8-OH-DPAT with those during what we scored as NREM after 8-OH-DPAT. Although neck EMG amplitude, EEG amplitude, and $\delta$ activity were all lower during NREM after 8-OH-DPAT compared with control NREM values, they all remained considerably higher than during REM before $8-\mathrm{OH}-$ DPAT $(p<0.03)$.

\section{8-OH-DPAT and the $5-\mathrm{HT}_{1 \mathrm{~A}}$ receptor}

To confirm that the effects on sleep were secondary to activation of $5-\mathrm{HT}_{1 \mathrm{~A}}$ receptors, we locally dialyzed WAY100635, a selective
B
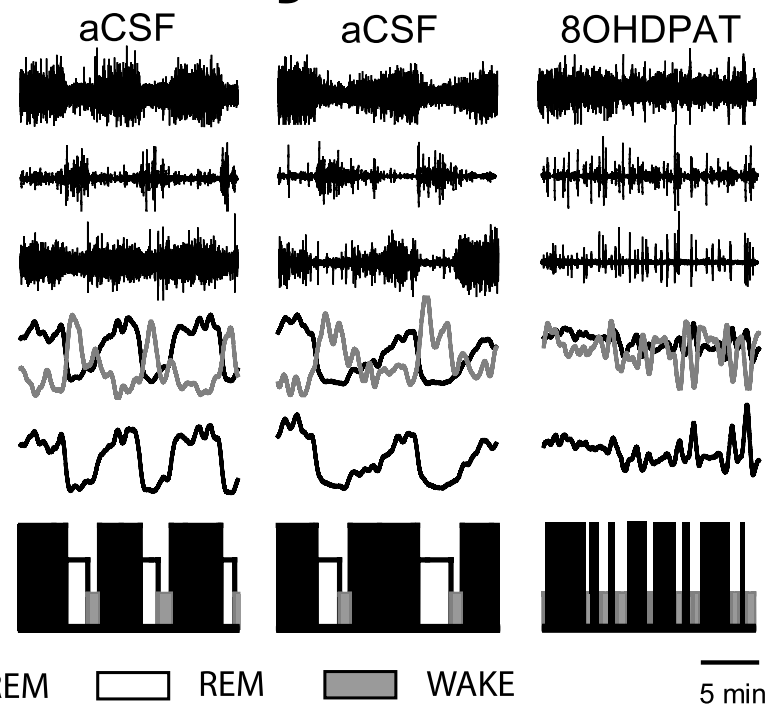

NREM

REM

$\overline{5 \min }$

Figure 3. A typical 8-OH-DPAT dialysis experiment. Data include EEG, EOG, neck EMG, delta or slow wave activity (SWA), the creased $n E M G$. REM is indicated by low EEG amplitude and SWA, an increased TDratio, rapid eye movements, and low neck EMG aCSF dialysis. B, 8-OH-DPAT experiment. Recordings were taken from the baseline period (first panel) and experimental period (second panel), during which 8-OH-DPAT was dialyzed into the PGCL.
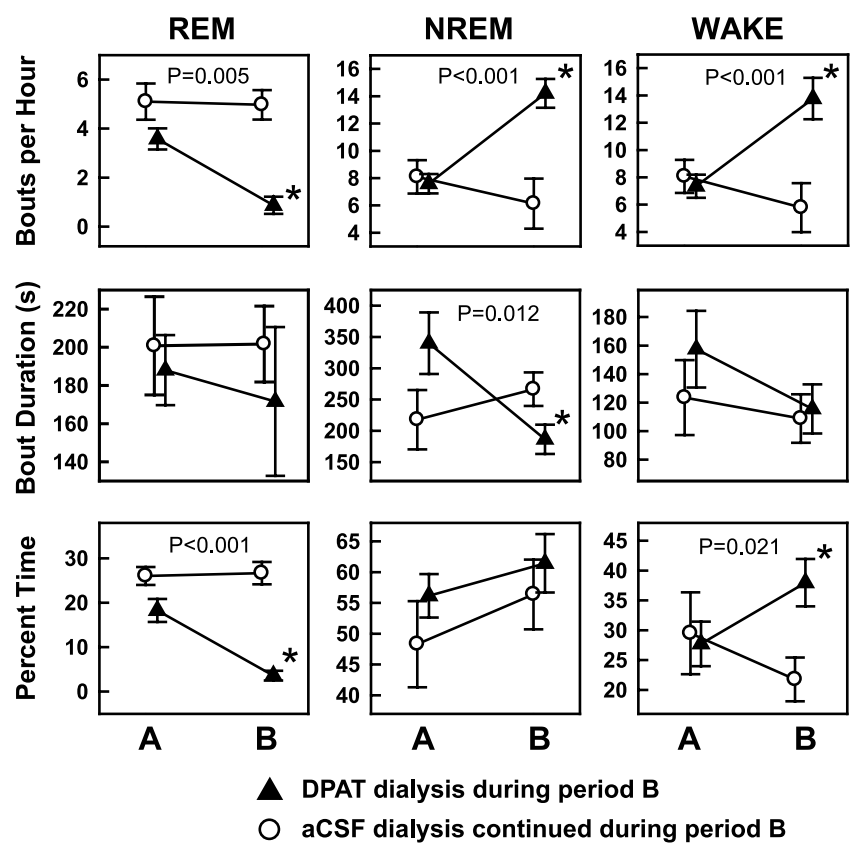

Figure 4. The effects of dialysis of 8-OH-DPAT (DPAT) into the PGCL on bout number per hour, bout duration, and the percentage of time spent in REM, NREM, and WAKE. In all panels, open circles are data from the control group $(n=6)$ in which aCSF was dialyzed during the entire experiment. Filled triangles are data from the 8-OH-DPAT group $(n=17)$ in which a period of aCSF dialysis was followed by a 30 min period of $8-0 \mathrm{H}-D P A T$ dialysis. The label " $A$ " on the $x$-axis represents the mean of values recorded during a $1 \mathrm{~h}$ baseline period during which both groups were dialyzed with aCSF. The label " $B$ " on the $x$-axis represents a $1 \mathrm{~h}$ experimental period during which the control group continued with aCSF dialysis and the 8-OH-DPAT group was dialyzed with 8-OH-DPAT. The differences between $A$ and $B$ in the control and 8-OH-DPAT groups were compared. An asterisk indicates a significant difference between the changes from $A$ to $B$ in the two groups. Error bars represent SEM. 

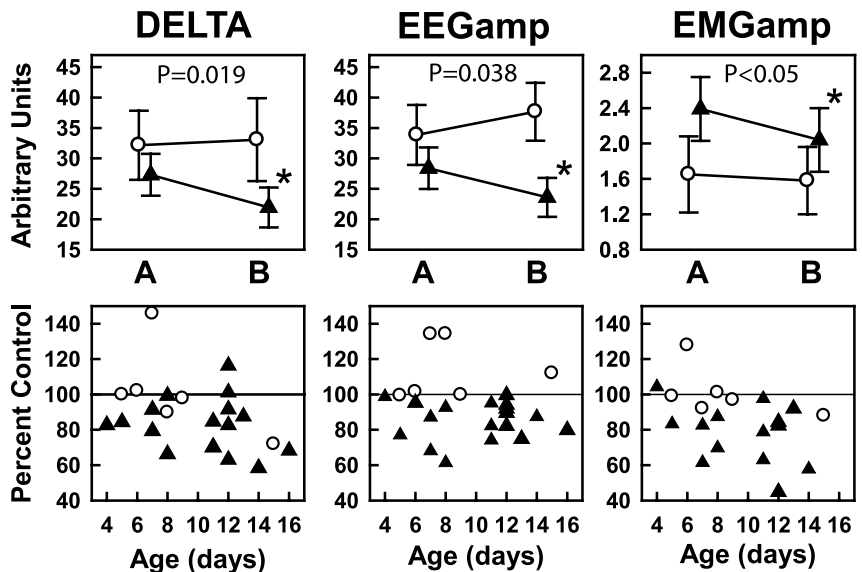

- DPAT dialysis during period $B$

$O$ aCSF dialysis continued during period $B$

Figure 5. The effect of 8-OH-DPAT (DPAT) on delta or slow wave activity, EEG amplitude (EEGamp), and neck EMG amplitude (EMGamp) during NREM sleep. For both the top and bottom panels, the control group $(n=6)$ is plotted as open circles, and the 8-OH-DPAT group $(n=$ 17) is plotted as filled triangles. Top, The label " $A$ " on the $x$-axis represents the mean of values recorded during a $1 \mathrm{~h}$ baseline period during which both groups were dialyzed with aCSF. The label " $\mathrm{B}$ " on the $x$-axis represents a $1 \mathrm{~h}$ experimental period during which the control group continued with aCSF dialysis, and the 8-OH-DPAT group was dialyzed with 8-OH-DPAT. The differences between $A$ and $B$ in the control and 8-OH-DPAT groups were compared. An asterisk indicates a significant difference between the changes from $A$ to $B$ in the two groups. Bottom, Individual data plotted according to age. Each point represents the difference between values during $A$ and $B$ from a single experiment, expressed as the percentage of the baseline period A. Error bars represent SEM.

$5-\mathrm{HT}_{1 \mathrm{~A}}$ receptor antagonist, into four animals before dialyzing 8-OH-DPAT. Figure 6 shows data from a single experiment. Note that sleep cycling is preserved during 8-OH-DPAT dialysis. The main effect of pretreatment with WAY100635, after taking age into account, was an inhibition of the effect of 8-OH-DPAT on REM bout number and percentage of time spent in REM. The ability of WAY100635 to prevent 8-OH-DPAT-induced changes in bout duration was less consistent. Figure 7 shows the group and individual data for bout number, comparing the WAY100635 data with both the control and $30 \mathrm{~mm} 8-\mathrm{OH}-\mathrm{DPAT}$ experiments. These data support the hypothesis that the effects of 8-OH-DPAT on sleep architecture are secondary to activation of $5-\mathrm{HT}_{1 \mathrm{~A}}$ receptors.

We also hypothesized that the effects that we observed after 8-OH-DPAT dialysis were secondary to activation of 5- $\mathrm{HT}_{1 \mathrm{~A}}$ receptors located on 5-HT neurons and functioning as autoreceptors. Although it is widely accepted that 5-HT neurons located in the raphé colocalize with $5-\mathrm{HT}_{1 \mathrm{~A}}$ receptors, there are no reports showing the presence of $5-\mathrm{HT}_{1 \mathrm{~A}}$ receptors located on the somata or dendrites of 5-HT neurons in the PGCL. Although we did not do a quantitative analysis, we did confirm colocalization of $\mathrm{TPOH}$-ir neurons and $5-\mathrm{HT}_{1 \mathrm{~A}}$ receptors using immunohistochemistry. It was estimated that $90 \%$ of TPOH-ir neurons on each section also contained label for $5-\mathrm{HT}_{1 \mathrm{~A}}$ receptors (D. S. Paterson, personal communication). The results from a representative section are shown in Figure 8. Examination of the distribution of the $5-\mathrm{HT}_{1 \mathrm{~A}}$ receptor immunoreactivity suggests that $5-\mathrm{HT}_{1 \mathrm{~A}}$ receptors are likely colocalized with both serotonergic and nonserotonergic neurons in the region of the PGCL that we studied.

To determine whether the effects that we observed were primarily secondary to activating autoreceptors, we unilaterally de-
WAY 100635 8-OH-DPAT

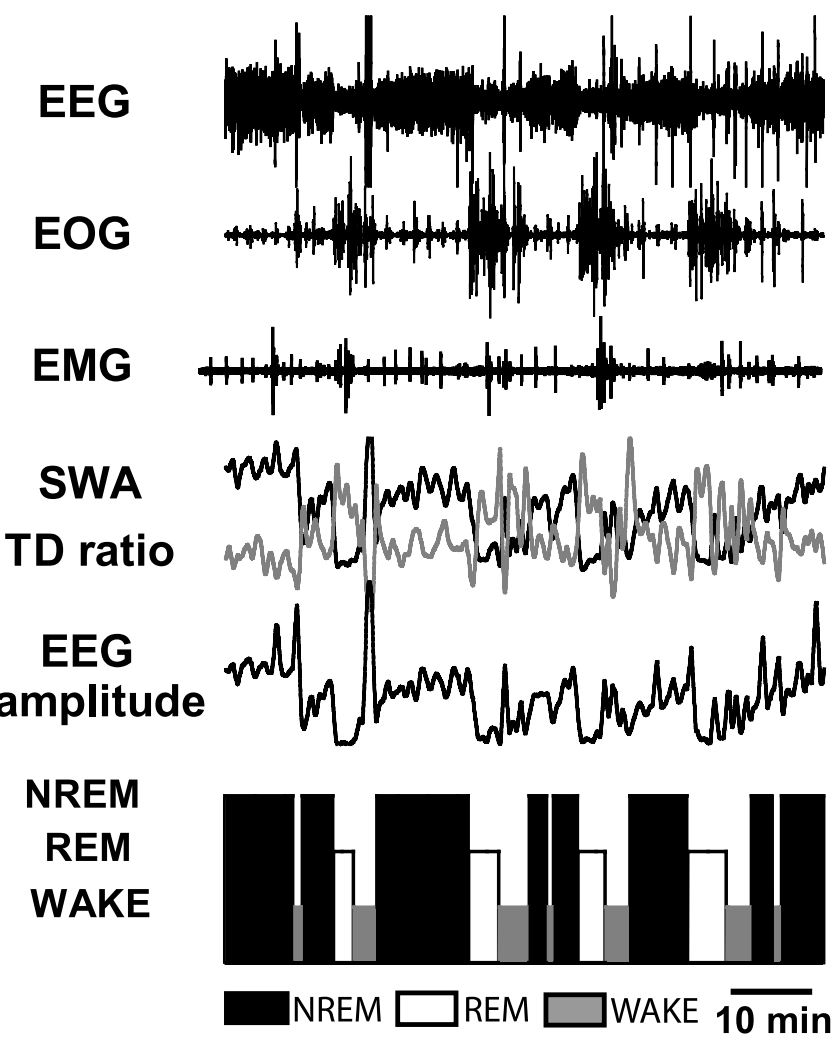

Figure 6. Recording from a single experiment in which WAY100635 was dialyzed before 8-OH-DPAT dialysis. Data include EEG, EOG, neck EMG, delta or slow wave activity (SWA), the TDratio, EEG amplitude, and a hypnogram showing NREM (black bars), REM (white bars), and WAKE (gray bars) periods. Periods of NREM are indicated by increases in EEG amplitude and SWA activity, no rapid eye movements, and relatively increased $n E M G$. REM is indicated by a low EEG amplitude and SWA, an increased TDratio, rapid eye movements, and a decrease in neck EMG. Transitions to WAKE are indicated by an abrupt increase in neck EMG, a drop in the TDratio, and a gradual rise in SWA and EEG amplitude. Note that sleep cycling is preserved and REM is not abolished after 8-OH-DPAT dialysis when pretreated with WAY100635.

stroyed 5-HT neurons in the PGCL in four piglets by dialyzing 5,7-DHT, a selective toxin for 5-HT neurons, after pretreatment with desipramine to minimize destruction of any nearby TyrOHcontaining neurons. After 1 week, 8-OH-DPAT was dialyzed into the regions, and the effects on sleep were evaluated and compared with the effects of 8-OH-DPAT dialysis in a subgroup of animals that were $\geq 12 \mathrm{~d}$ of age. Figure 9 illustrates the destruction of 5-HT neurons secondary to 5,7-DHT dialysis in one animal. Adjacent sections were stained with cresyl violet (Fig. 9A), for TPOH immunoreactivity (Fig. 9B), and for $\mathrm{TyrOH}$ immunoreactivity (Fig. 9C). Note the large area devoid of TPOH-ir neurons on the lesioned side (Fig. 9B). Also, there are very few TyrOHimmunoreactive (TyrOH-ir) neurons in this region, most lying more lateral to the rostral portion of the PGCL and importantly, as shown in Figure 9C, unaffected by 5,7-DHT. The cell counts are shown in Figure 10, showing the four individual piglets as well as the averaged data. The individual data show the location of the lesion in reference to the caudal pole of the facial nucleus, whereas the averaged data are referenced to the caudal edge of the lesion. The number of 5-HT neurons destroyed, expressed as a percentage of the control (nonlesioned) side, ranged from 39.7 to $77.5 \%$ and averaged $64.6 \pm 8.7 \%$.

By 1 week after 5,7-DHT dialysis, sleep was more fragmented 


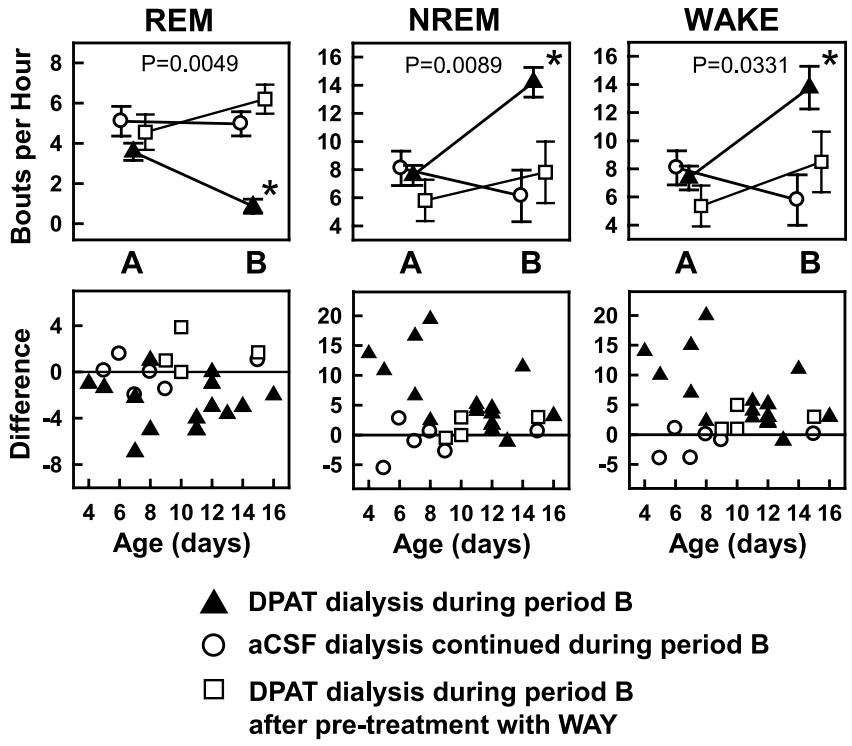

Figure 7. Mean and individual bout number per hour data for REM, NREM, and WAKE from experiments in which WAY100635 was dialyzed before dialysis of 8-0H-DPAT (DPAT). Mean data from WAY100635 plus 8-OH-DPAT experiments (open squares; $n=4$ ), control experiments in which aCSF was dialyzed over the entire study period (open circles; $n=6$ ), and 8-OH-DPAT experiments in which aCSF dialysis was followed by 8-OH-DPAT dialysis (filled triangles; $n=17$ ) are shown on the top panel. The label " $\mathrm{A}$ " on the $x$-axis represents the mean of values recorded during a $1 \mathrm{~h}$ baseline period during which all groups were dialyzed with aCSF. The label " $\mathrm{B}$ " on the $x$-axis represents a $1 \mathrm{~h}$ experimental period during which the control group continued with aCSF dialysis, the 8-OH-DPAT group was dialyzed with 8-OH-DPAT, and the WAY100635 plus 8-0H-DPAT group was first dialyzed with WAY100635 followed by 8-OH-DPAT dialysis. The differences between A and B in the control, 8-OH-DPAT, and WAY100635 plus 8-OH-DPAT groups were compared. An asterisk indicates a significant difference between the changes from A to $B$ the 8-0H-DPAT group and the WAY100635 plus 8-0H-DPAT group. The control, 8-OH-DPAT, and WAY100635 plus 8-0H-DPAT groups are shown in the bottom panel plotted against age. Each point represents the difference between values during $A$ and $B$ for a single experiment. Error bars represent SEM.

compared with similar aged animals. There were more $(p=$ $0.023)$ and shorter $(p<0.05)$ bouts of NREM per hour, with little or no change in the percentage of time spent in state. The bout number per hour, or bout duration of REM, was not different, and the percentage of time spent in REM was not affected. However, subsequent dialysis of 8-OH-DPAT did not decrease the amount of REM or change the relationship between the number, or duration, of bouts of WAKE or NREM, compared with a control group of similar ages (Fig. 11). Interestingly, the effect of 8-OH-DPAT on body temperature was not different in the animals treated with 5,7-DHT, compared with the control group of similar ages. Similarly, HR decreased after 8-OH-DPAT dialysis in the 5,7-DHT-treated animals similar to the changes in a similar-aged control group. These data indicate that the effects on sleep that we observed after 8-OH-DPAT dialysis were secondary to activation of somato-dendritic $5-\mathrm{HT}_{1 \mathrm{~A}}$ autoreceptors. However, the effects of 8-OH-DPAT on body temperature and HR may have been secondary to stimulating postsynaptic $5-\mathrm{HT}_{1 \mathrm{~A}}$ receptors located on nonserotonergic neurons that were involved in modulating these variables, perhaps by affecting sympathetic outflow.

Effect of 8-OH-DPAT on other physiological variables

$\mathrm{CO}_{2}$ concentration in the plethysmograph did not change over the course of the experiments and averaged $\sim 0.42-0.48 \%$. Values for cardio-respiratory variables were related to age. Under control conditions, $V_{\mathrm{E}}$ decreased with age during all states, pri- marily because of a decrease in RR with little or no change in $V_{\mathrm{T}}$. Heart rate, but not BPm, also decreased with age. Cardiorespiratory variables were modulated by state, confirming what we have reported previously (Darnall et al., 2001). Because of the marked decrease in the amount of REM after 8-OH-DPAT dialysis, we were not able to perform a complete analysis on the effects of 8-OH-DPAT dialysis. However, there were no consistent effects of 8-OH-DPAT dialysis on RR, $V_{\mathrm{T}}$, or $V_{\mathrm{E}}$ during NREM or WAKE. In contrast, 8-OH-DPAT dialysis caused HR to decrease in 11 of 13 animals $(p=0.01)$. The mean decrease in HR was $7.7 \pm 2.7$ and $8.2 \pm 2.9$ beats $/ \mathrm{min}$ for WAKE and NREM, respectively. Although there was a small increase in BPm in 10 of 13 animals, the overall effect was not significant $(p=0.07)$.

The wall temperature of the plethysmograph was adjusted in an attempt to achieve a thermoneutral environment based on weight and age. As a result, the air temperatures inside the plethysmograph varied with weight $(p=0.017)$, being lowest with the animals of greatest weight, but did not change over the course of the experiment (Table 1). Body temperature was related to both age and weight, and after taking this into account, body temperature fell after 8-OH-DPAT dialysis and was more pronounced in the younger animals (Table 1). Because of the gas flow dynamics of the plethysmograph, it was not possible to accurately measure changes in $\mathrm{VO}_{2}, \mathrm{VCO}_{2}$, and RQ across state changes. All states were therefore combined to assess the relationship to age and the effect of 8-OH-DPAT dialysis. $\mathrm{VCO}_{2}(p=$ $\left.0.001 ; R^{2}=0.24\right)$, but not $\mathrm{VO}_{2}$, decreased with increasing age resulting in a decreasing RQ $\left(p=0.044 ; R^{2}=0.08\right) .8-\mathrm{OH}-$ DPAT dialysis did not result in any consistent changes in $\mathrm{VO}_{2}$, $\mathrm{VCO}_{2}$, or RQ. The mean values for $\mathrm{VO}_{2}, \mathrm{VCO}_{2}$, and RQ before and after sham aCSF dialysis and before and after 8-OH-DPAT dialysis are shown in Table 1.

\section{Discussion}

The major findings are that dialyzing 8-OH-DPAT into the juxtafacial PGCL causes sleep fragmentation and a marked decrease in the amount of REM sleep. Although the rostral groups of 5-HT neurons play important roles in the regulation of sleep (Portas et al., 1996; Strecker et al., 1999; Monti et al., 2000; Sakai and Crochet, 2000; Sorensen et al., 2001), little is known about the role of 5-HT neurons in the PGCL in either regulating or modulating sleep. Large bilateral quisqualic acid lesions of the medial reticular formation encompassing the juxtafacial PGCL decrease the amount of REM (Holmes and Jones, 1994). Nonspecific neuronal inhibition with $\mathrm{GABA}_{\mathrm{A}}$ agonists (Curran et al., 2001; Darnall et al., 2001; Messier et al., 2002) or lidocaine (Berner et al., 1999) in this region decreases the amount of sleep and increases wakefulness. In studies focusing on the role of 5-HT neurons in the midline raphé and immediate adjacent areas, dialysis or microinjection of 8-OH-DPAT decreases sleep and increases wakefulness (Berner et al., 1999; Messier et al., 2004). We report the novel finding that 5-HT neurons in the juxtafacial PGCL play a role in the modulation of sleep and in particular, REM sleep. These findings suggest a possible link between sleep homeostasis and the abnormalities in serotonergic receptor binding found in a large subset of SIDS infants in two large independent data sets.

\section{The sleep effects of 8-OH-DPAT dialysis into the}

PGCL are caused by activation of $5-\mathrm{HT}_{1 \mathrm{~A}}$ somato-dendritic autoreceptors

It is likely that both somato-dendritic autoreceptors and postsynaptic $5-\mathrm{HT}_{1 \mathrm{~A}}$ receptors located on non-5-HT neurons were activated by 8-OH-DPAT in our studies (Thor et al., 1992; Kia et al., 

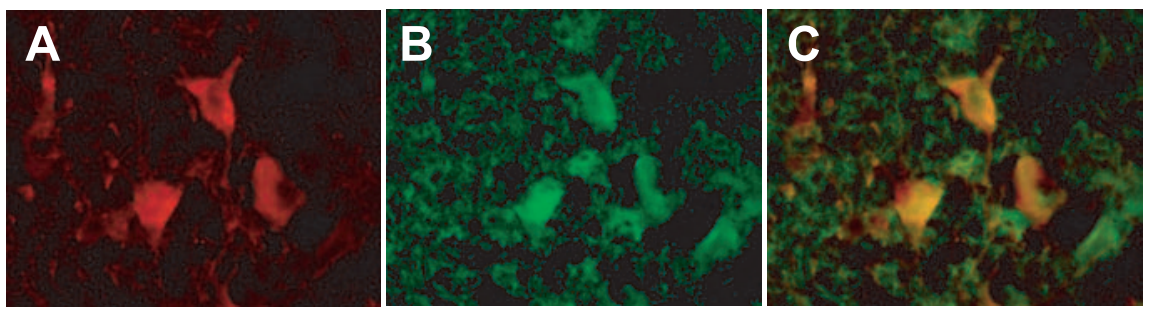

Figure 8. Double labeling of TPOH-ir neurons and 5- $\mathrm{HT}_{1 \mathrm{~A}}$-immunoreactive receptors in one section of the $\mathrm{PGCL}$ near the caudal pole of the facial nucleus. $\boldsymbol{A}, \mathrm{TPOH}-$ ir neurons are labeled in red. $\boldsymbol{B}, 5-\mathrm{HT} 1 \mathrm{~A}$ receptors are labeled in green. $\boldsymbol{C}, 0$ verlay of $\boldsymbol{A}$ and $\boldsymbol{B}$.
(Niblock et al., 2004), (2) most of these $(\sim 90 \%)$ colocalize with $5-\mathrm{HT}_{1 \mathrm{~A}}$ receptors, (3) pretreatment with the selective $5-\mathrm{HT}_{1 \mathrm{~A}}$ antagonist WAY100635 abolishes the sleep disrupting effects of subsequent $8-\mathrm{OH}-$ DPAT dialysis, and (4) dialysis of 5,7-DHT into the PGCL produces sleep fragmentation similar to that produced with $8-\mathrm{OH}$ DPAT dialysis and eliminates the effect of subsequent 8-OH-DPAT dialysis on REM.

The effects of 8-OH-DPAT dialysis on body temperature and heart rate were
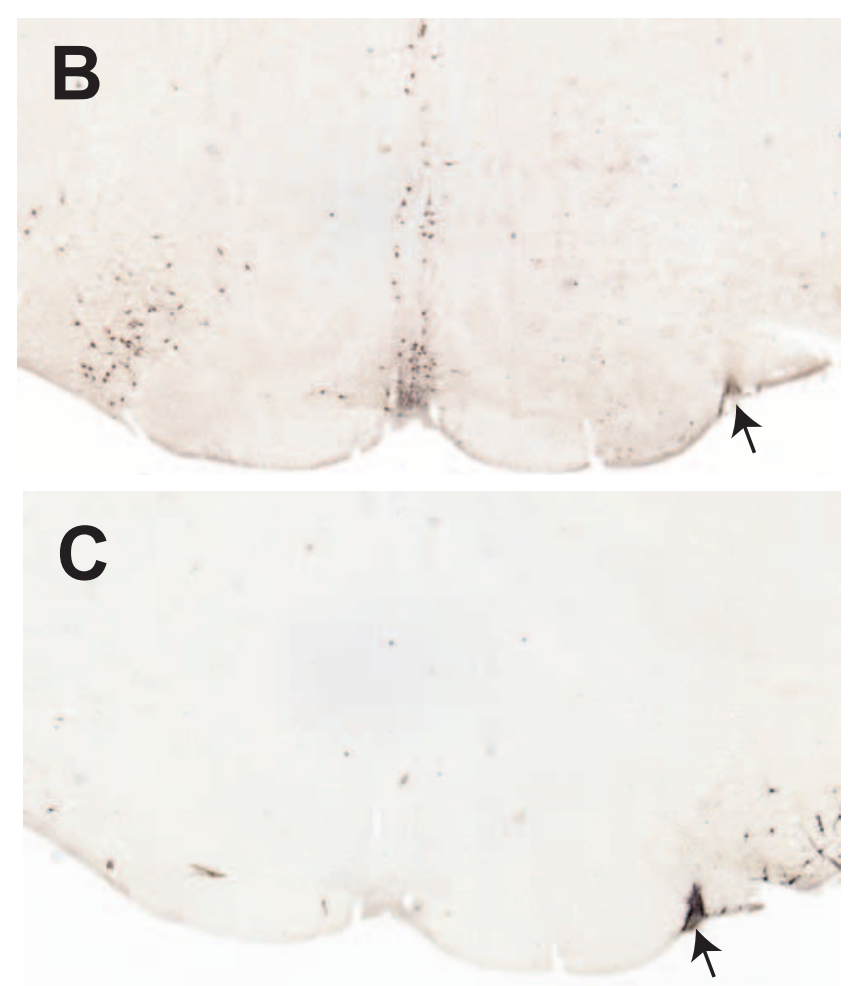

Figure 9. Unilateral destruction of TPOH-ir neurons on with 5,7-DHT. A, Standard Nissl stain (cresyl violet) showing many neurons scattered throughout the area. $\boldsymbol{B}$, Section stained for TPOH-ir neurons. Note the absence of neurons on the lesioned (right) side. C, Section stained for TyrOH-ir neurons. Note the scarcity of TyrOH-ir neurons in the PGCL on either side with their location limited to the very lateral edges of the section. Arrows point to the dialysis probe tip location. GC, Gigantocellularis; ROb, raphé obscurus; RPal, raphé pallidus; Pyr, pyramid; ION, inferior olive.

1996). However, the primary effects on sleep were caused by activation of $5-\mathrm{HT}_{1 \mathrm{~A}}$ somato-dendritic autoreceptors. This conclusion is based on our results showing that (1) there are large numbers of 5-HT neurons located in the PGCL in the piglet likely attributable to the activation of postsynaptic $5-\mathrm{HT}_{1 \mathrm{~A}}$ receptors. 5- $\mathrm{HT}_{1 \mathrm{~A}}$ receptors localize to nonserotonergic neurons in the raphé pallidus and parapyramidal region (Morrison, 2004) and in the rostral ventrolateral medulla (Holmes et al., 1994; Helke et al., 1997), where they are involved in thermoregulation and the control of blood pressure, respectively. Most of these neurons project to the intermediolateral column of the spinal cord and influence sympathetic activity involved in thermoregulation and cardiovascular control (Guyenet, 1990; Helke et al., 1997; Nakamura et al., 2004). Local application of 8-OH-DPAT into these regions, including the PGCL, in anesthetized animals consistently decreases blood pressure (Lovick, 1989; Helke et al., 1993). In contrast, we found in the conscious piglet that local dialysis of 8-OH-DPAT into the PGCL causes little or no change in blood pressure and a decrease in heart rate. These results are similar to those reported in two other studies in conscious animals in which 8-OH-DPAT was either dialyzed or microinjected into the caudal raphé and caused a decrease in heart rate with little or no change in blood pressure (Messier et al., 2004; Nalivaiko et al., 2005). Furthermore, destruction of 5-HT neurons with 5,7-DHT did not prevent the effects of subsequent 8-OH-DPAT dialysis on body temperature or heart rate, supporting the hypothesis that these effects were mediated by activation of postsynaptic $5-\mathrm{HT}_{1 \mathrm{~A}}$ receptors located on nonserotonergic neurons.

\section{Activation of 5- $\mathrm{HT}_{1 \mathrm{~A}}$ autoreceptors in the PGCL decreases the amount of REM sleep}

A decrease in REM after 8-OH-DPAT dialysis in the PGCL was a consistent finding in all animals. Why does activation of $5-\mathrm{HT}_{1 \mathrm{~A}}$ autoreceptors in this region of the brainstem decrease REM sleep? One possibility is that sleep fragmentation after 8-OH-DPAT dialysis prevented the development of REM (Benington and Heller, 1994; Borbely, 1994). However, destruction of 5-HT neurons with 5,7-DHT produced the same level of sleep fragmentation without affecting the amount of REM suggesting that these may be independent processes.

We postulate that the decrease in REM sleep after 8-OHDPAT dialysis was secondary to the critical role played by $5-\mathrm{HT}$ neurons in the PGCL in integrating multiple sensory inputs and modulating brain arousal and alerting systems. Neurons in the PGCL have major projections to the LC (Van Bockstaele and Aston-Jones, 1995), where there is a dense innervation of serotonergic fibers and terminals (Pickel et al., 1977). Serotonin attenuates excitatory amino acid responses of LC neurons (AstonJones et al., 1991b). The ventral oral pontine reticular nucleus, a region important for the generation and maintenance of REM sleep, receives abundant serotonergic innervation that is not from the dorsal or median raphé (Rodrigo-Angulo et al., 2000) and may include those from the PGCL. Thus, it is reasonable to hypothesize that withdrawal of serotonergic input to the LC and 

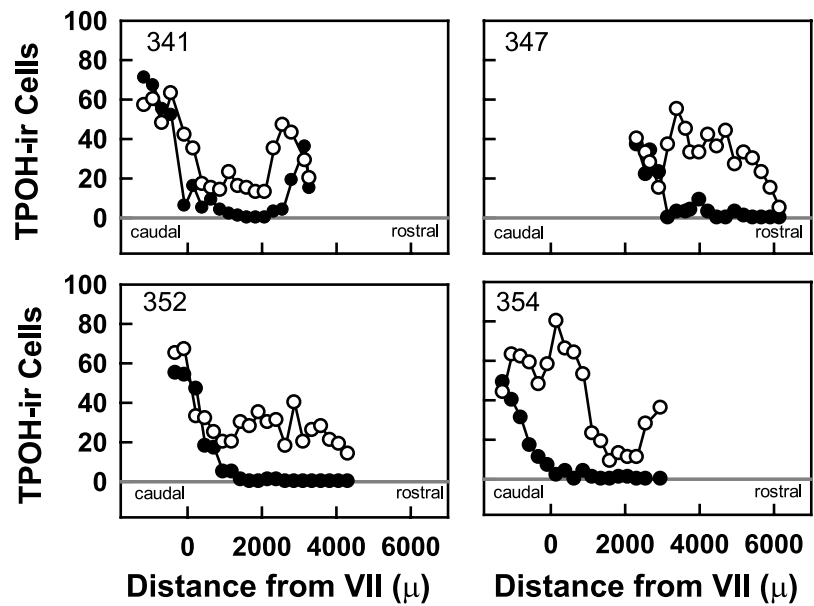

0200040006000
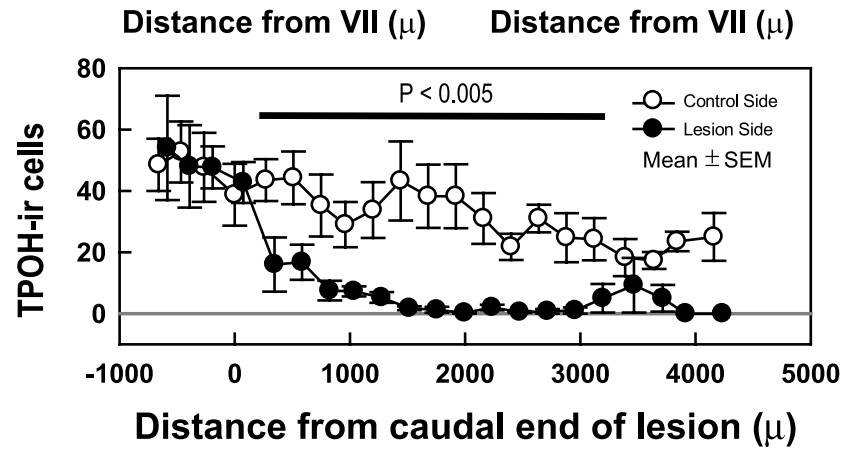

Figure 10. Cell counts showing the number of TPOH-ir neurons destroyed with 5,7-DHT on the lesioned side compared with the nonlesioned side. The top four panels show the counts from the individual animals referenced to the caudal pole of the facial nucleus (VII) to show the actual location of each lesion. Each set of points represent counts from one $40 \mu \mathrm{m}$ section. Approximately every sixth section was counted. Open circles are counts from the unlesioned side, and filled circles are counts from the lesioned side. The bottom panel shows the group counts expressed as mean \pm SEM referenced to the caudal end of the lesion.

surrounding regions might interrupt the normal state related attenuation leading to alterations in REM sleep homeostasis.

Serotonergic neurons in the PGCL also have major projections to the dorsal horn of the spinal cord (Skagerberg and Bjorklund, 1985; Kwiat and Basbaum, 1992), where they modulate nociceptive (Basbaum and Fields, 1984; Leung and Mason, 1999) and non-nociceptive (Gray and Dostrovsky, 1983, 1985) sensory inputs. Furthermore, 5,6-DHT spinal cord lesions decrease REM in rats (Bjorkum et al., 1995), and intrathecal administration of 8-OH-DPAT increases the amount of sleep and decreases the amount of wakefulness (Bjorkum and Ursin, 1996). These data support the hypothesis that 5-HT neurons in the PGCL can modulate sleep state by dampening sensory input at the spinal cord level. Thus, inhibition of the activity of these neurons could result in an "undampening" of ascending sensory information promoting more wakefulness and the sleep fragmentation that we observed.

\section{Dialysis of 8-OH-DPAT into the PGCL decreases muscle tone} during NREM sleep

Skeletal muscle hypotonia during NREM similar to that seen during REM under control conditions was observed in some animals after 8-OH-DPAT dialysis. In a few animals, an attenuation in shivering during NREM was also observed, similar what occurs during REM under control conditions (Parmeggiani and Rabini, 1967). The classic studies by Magoun and Rhines suggested that neurons in the caudal medulla are involved in the modulation of
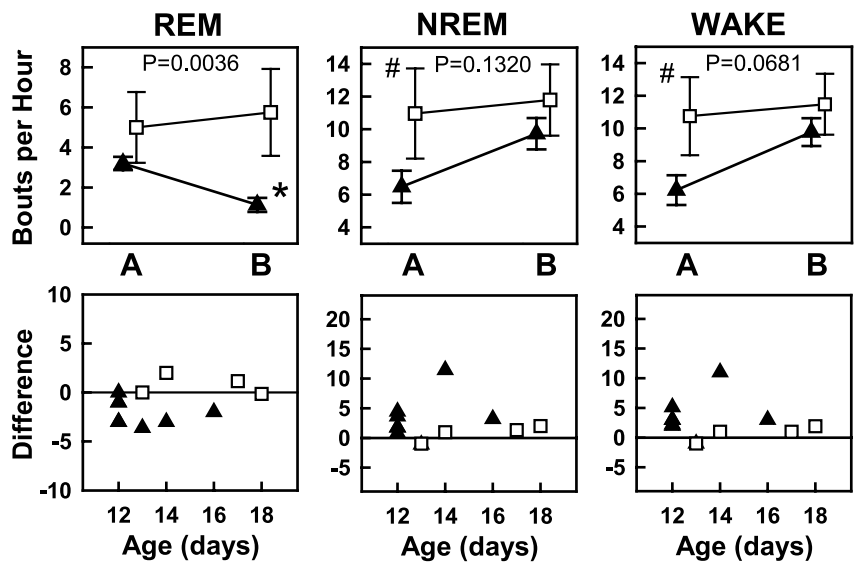

$\triangle$ DPAT dialysis during period B
$\square$ DPAT dialysis during period B
1 week after DHT dialysis.

Figure 11. Mean and individual data for bout number per hour for animals dialyzed with 5,7-DHT (DHT) and subsequently dialyzed with 8-OH-DPAT (DPAT) 1 week later compared with a group of animals of similar ages dialyzed with 8-OH-DPAT. The top panel shows the mean data. Open squares are the 5,7-DHT-treated animals $(n=4)$, and the filled triangles are a subgroup of 8-OH-DPAT experiments in which the animals were $\geq 12 \mathrm{~d}$ of age $(n=6)$. The label " $A$ " on the $x$-axis represents the mean of values recorded during a $1 \mathrm{~h}$ baseline period during which both groups were dialyzed with aCSF. The label " $B$ " on the $x$-axis represents a $1 \mathrm{~h}$ experimental period during which both the 5,7-DHT-treated and nontreated animals were dialyzed with 8-OH-DPAT. The differences between A and B in the 5,7-DHT-treated and nontreated groups were compared. An asterisk indicates that there is a significant difference between the changes in the two groups. In addition, \# indicates that the baseline values were different in the two groups. Error bars represent SEM.

Table 1. The effects of 8-OH-DPAT dialysis on selected metabolic variables

\begin{tabular}{llllll}
\hline & \multicolumn{2}{l}{ Control group } & & \multicolumn{2}{l}{ Experimental group } \\
\cline { 2 - 3 } \cline { 5 - 6 } Variable & aCSF & aCSF & & aCSF & 8-OH-DPAT \\
\hline $\mathrm{VO}_{2}\left(\mathrm{ml} \cdot \mathrm{kg}^{-1} \cdot \mathrm{min}^{-1}\right)$ & $16.0 \pm 0.8$ & $15.3 \pm 0.9$ & & $16.3 \pm 0.4$ & $15.4 \pm 0.5$ \\
$\mathrm{VCO}_{2}\left(\mathrm{ml} \cdot \mathrm{kg}^{-1} \cdot \mathrm{min}^{-1}\right)$ & $13.3 \pm 0.6$ & $13.9 \pm 1.0$ & & $12.9 \pm 0.5$ & $13.2 \pm 0.8$ \\
$\mathrm{RQ}$ & $0.96 \pm 0.05$ & $1.15 \pm 0.12$ & & $0.89 \pm 0.04$ & $0.88 \pm 0.10$ \\
Air temperature $\left({ }^{\circ} \mathrm{C}\right)$ & $26.6 \pm 0.6$ & $26.7 \pm 0.4$ & & $26.3 \pm 0.3$ & $26.4 \pm 0.3$ \\
Body temperature $\left({ }^{\circ} \mathrm{C}\right)$ & $38.4 \pm 0.2$ & $38.5 \pm 0.3$ & & $38.7 \pm 0.2$ & $38.2 \pm 0.2^{*}$ \\
\hline
\end{tabular}

Data include $\mathrm{VO}_{2}, \mathrm{VCO}_{2}, \mathrm{RQ}$, and plethysmograph air temperature and piglet core body temperature. Dialysis of 8-OH-DPAT caused a small but significant $\left(^{*}\right)$ decrease in body temperature.

muscle tone (Magoun, 1944; Magoun and Rhines, 1946). Neurons in PGCL project to thoracic, lumbar, and sacral segments of the spinal cord (Kausz, 1991) and have axon collateral projections to both autonomic and somatic cell groups (Allen and Cechetto, 1994). Spinally projecting glycinergic neurons that receive glutamatergic, cholinergic, and orexinergic inputs in this region have been implicated in the hypotonia associated with REM (Kodama et al., 1998; Lai et al., 1999; Hajnik et al., 2000; Mileykovskiy et al., 2002), and some appear to be serotonergic (Fort et al., 1993; Stornetta et al., 2004). It is possible that activation of either $5-\mathrm{HT}_{1 \mathrm{~A}}$ somato-dendritic receptors on glycinergic/ 5-HT neurons or postsynaptic receptors located on non-5-HT glycinergic neurons might result in a change in muscle tone.

What we scored as short periods NREM after 8-OH-DPAT dialysis might represent a dissociated state with REM-like hypotonia. However, there were no rapid eye movements or REM-like changes in respiration, blood pressure, and heart rate. In addition, although the levels of $\delta$ power, EEG amplitude, and neck EMG activity were lower during NREM after 8-OH-DPAT dialysis, they were significantly higher than during REM bouts before 
8-OH-DPAT. We therefore believe that the relative hypotonia after 8-OH-DPAT dialysis into the PGCL is more likely related to the role of 5-HT neurons of providing tonic state related motor facilitation important for the modulation of muscle tone and rhythmic motor activity (Jacobs and Fornal, 1999).

\section{Implications for sudden infant death syndrome}

Victims of sudden infant death syndrome presumably die during sleep. However, there has been little evidence to support a specific sleep abnormality. The focus of research has been on protective arousal mechanisms that help defend against stressors often encountered during sleep. However, an increase in arousability may not always be an advantage because it might lead to fragmented sleep and sleep deprivation. Prolonged deep periods of recovery sleep might also increase the risk of obstructive sleep apnea (Simpson, 2001). REM sleep deprivation in adult humans and animals has been found to cause many behavioral changes (Dement, 1960; Koller et al., 1969), and brief periods of sleep deprivation in infants alters the autonomic control of heart rate (Franco et al., 2003) and decrease arousals (Franco et al., 2004). Our new findings suggest that abnormalities in medullary serotonergic neurons produce sleep fragmentation and decreased amounts of REM sleep. $5-\mathrm{HT}_{1 \mathrm{~A}}$ receptor activation in this study was restricted to a region where abnormal serotonergic receptor binding has been demonstrated in SIDS infants. More widespread serotonergic receptor dysfunction may lead to considerable alterations in sleep homeostasis, increasing the risk for sudden death.

\section{References}

Allen GV, Cechetto DF (1994) Serotoninergic and nonserotoninergic neurons in the medullary raphé system have axon collateral projections to autonomic and somatic cell groups in the medulla and spinal cord. J Comp Neurol 350:357-366.

Andrezik JA, Chan-Palay V, Palay SL (1981) The nucleus paragigantocellularis lateralis in the rat: conformation and cytology. Anat Embryol 161:355-371.

Aston-Jones G, Ennis C, Pieribone VA, Nickell WT, Shipley MT (1986) The brain nucleus locus coeruleus: restricted afferent control of a broad efferent network. Science 234:734-737.

Aston-Jones G, Shipley MT, Chouvet G, Ennis M, van Bockstaele E, Pieribone V, Shiekhattar R, Akaoka H, Drolet G, Astier B, Charléty P, Valentino RJ, Williams JT (1991a) Afferent regulation of locus coeruleus neurons: Anatomy, physiology and pharmacology. Prog Brain Res 88:47-75.

Aston-Jones G, Akaoka H, Chariety P, Chouvet G (1991b) Serotonin selectively attenuates glutamate-evoked activation of noradrenergic locus coeruleus neurons. J Neurosci 11:760-769.

Bartlett D, Tenney SM (1970) Control of breathing in experimental anemia. Respir Physiol 10:384-395.

Basbaum AI, Fields HL (1984) Endogenous pain control systems: brainstem spinal pathways and endorphin circuitry. Annu Rev Neurosci 7:309-338.

Benington JH, Heller HC (1994) REM-sleep timing is controlled homeostatically by accumulation of REM-sleep propensity during non-REM sleep. Am J Physiol 266:R1992-R2000.

Berner NJ, Grahn DA, Heller HC (1999) 8-OH-DPAT-sensitive neurons in the nucleus raphe magnus modulate thermoregulatory output in rats. Brain Res 831:155-164.

Bjorkum AA, Ursin R (1996) Sleep/waking effects following intrathecal administration of the 5-HT1A agonist 8-OH-DPAT alone and in combination with the putative 5-HT1A antagonist NAN-190 in rats. Brain Res Bull 39:373-379.

Bjorkum AA, Neckelmann D, Bjorvatn B, Ursin R (1995) Lesion of descending 5-HT pathways increases zimeldine-induced waking in rats. Physiol Behav 57:959-966.

Borbely AA (1994) Sleep homeostasis and models of sleep regulation. In: Principles and practice of sleep medicine, Ed 2 (Kryger M, Roth T, Dement WC, eds), pp 309-320. Philadelphia: W. B. Saunders Company.

Bowker RM, Abbott LC (1990) Quantitative re-evaluation of descending serotonergic and nonserotonergic projections from the medulla of the rodent: evidence for extensive co-existence of serotonin and peptides in the same spinally projecting neurons, but not from the nucleus raphe magnus. Brain Res 512:15-25.

Curran AK, Chen G, Darnall RA, Filiano JJ, Li A, Nattie EE (2000) Lesions or muscimol in the rostral ventral medulla reduces ventilatory output and the $\mathrm{CO}_{2}$ response in decerebrate piglets. Respir Physiol 123:23-37.

Curran AK, Darnall RA, Filiano JJ, Li A, Nattie EE (2001) Muscimol dialysis in the rostral ventral medulla reduces the $\mathrm{CO} 2$ response in awake and sleeping piglets. J Appl Physiol 90:971-980.

Darnall RA, Curran AK, Filiano JJ, Li A, Nattie EE (2001) The effects of a $\mathrm{GABA}_{\mathrm{A}}$ agonist in the rostral ventral medulla on sleep and breathing in newborn piglets. Sleep 24:514-527.

De Lange EC, Bouw MR, Mandema JW, Danhof M, de Boer AG, Breimer DD (1995) Application of intracerebral microdialysis to study regional distribution kinetics of drugs in rat brain. Br J Pharm 116:2538-2544.

Dement WC (1960) Effect of dream deprivation. Science 131:1705-1707.

Drorbaugh JE, Fenn WO (1955) A barometric method for measuring ventilation in newborn infants. Pediatrics 16:81-87.

Filiano JJ, Kinney HC (1994) A perspective on neuropathologic findings in victims of the sudden infant death syndrome: the triple-risk model. Biol Neonate 65:194-197.

Fort P, Luppi PH, Jouvet M (1993) Glycine-immunoreactive neurones in the cat brain stem reticular formation. NeuroReport 4:1123-1126.

Franco P, Seret N, Van Hees JN, Lanquart JP, Grosswasser J, Kahn A (2003) Cardiac changes during sleep in sleep-deprived infants. Sleep 26:845-848.

Franco P, Seret N, Van Hees JN, Scaillet S, Vermeulen F, Groswasser J, Kahn A (2004) Decreased arousals among healthy infants after short-term sleep deprivation. Pediatrics 114:e192-e197.

Gray BG, Dostrovsky JO (1983) Descending inhibitory influences from periaqueductal gray, nucleus raphé magnus, and adjacent reticular formation. I. Effects on lumbar spinal cord nociceptive and non-nociceptive neurons. J Neurophysiol 49:932-947.

Gray BG, Dostrovsky JO (1985) Inhibition of feline spinal cord dorsal horn neurons following electrical stimulation of nucleus paragiganteocellularis lateralis. A comparison with nucleus raphé magnus. Brain Res 348:261-273.

Guyenet PG (1990) Role of the ventral medulla oblongata in blood pressure regulation. In: Central regulation of autonomic functions (Loewy AD, ed), p 168. New York: Oxford UP.

Hajnik T, Lai YY, Siegel JM (2000) Atonia-related regions in the rodent pons and medulla. J Neurophysiol 84:1942-1948.

Helke CJ, McDonald CH, Phillips ET (1993) Hypotensive effects of 5-HT1A receptor activation: ventral medullary sites and mechanisms of action in the rat. J Auton Nerv Syst 42:177-188.

Helke CJ, Capuano S, Tran N, Zhuo H (1997) Immunocytochemical studies of the 5-HT1A receptor in ventral medullary neurons that project to the intermediolateral cell column and contain serotonin or tyrosine hydroxylase immunoreactivity. J Comp Neurol 379:261-270.

Heym J, Steinfels GF, Jacobs BL (1982a) Medullary serotonergic neurons are insensitive to 5-MeoDMT and LSD. Eur J Pharm 81:677-680.

Heym J, Steinfels GF, Jacobs BL (1982b) Activity of serotonin-containing neurons in the nucleus raphé pallidus of freely moving cats. Brain Res 251:259-276

Holmes CJ, Jones BE (1994) Importance of cholinergic, GABAergic, serotonergic and other neurons in the medial medullary reticular formation for sleep-wake states studied by cytotoxic lesions in the cat. Neuroscience 62:1179-1200.

Holmes CJ, Mainville LS, Jones BE (1994) Distribution of cholinergic, GABAergic and serotonergic neurons in the medial medullary reticular formation and their projections studied by cytotoxic lesions in the cat. Neuroscience 62:1155-1178.

Holstege G, Kuypers HGJM (1987) Brainstem projections to spinal motoneurons: an update. Neuroscience 23:809-821.

Jacobs BL, Fornal CA (1999) Physiology and pharmacology of brain sertonergic neurons. In: Serotonergic neurons and 5-HT receptors in the CNS (Baumbgarten HG, Gothert M, eds), pp 91-116. Berlin: Springer.

Kahn A, Grosswasser J, Franco P, Scaillet S, Sawaguchi T, Kelmanson I, Bernanrd D (2002) Sudden infant deaths: arousal as a survival mechanism. Sleep Med 3:S11-S14.

Kausz M (1991) Arrangement of neurons in the medullary reticular forma- 
tion and raphe nuclei projecting to thoracic, lumbar and sacral segments of the spinal cord in the cat. Anat Embryol 183:151-163.

Kia HK, Miquel M-C, Brisorgueil M-J, Daval G, Riad M, Mestikawy SE, Hamon M, Verge D (1996) Immunocytochemical localization of serotonin1A receptors in the rat central nervous system. J Comp Neurol 365:289-305.

Kinney HC, Filiano JJ, Sleeper LA, Mandell F, Valdes-Dapena M, White WF (1995) Decreased muscarinic receptor binding in the arcuate nucleus in sudden infant death syndrome. Science 269:1446-1450.

Kinney HC, Randall LL, Sleeper LA, Willinger M, Belliveau RA, Zec N, Rava LA, Dominici L, Iyasu S, Randall B, Habbe D, Wilson H, Mandell F, McClain M, Welty TK (2003) Serotonergic brainstem abnormalities in Northern Plains Indians with the sudden infant death syndrome. J Neuropathol Exp Neurol 62:1178-1191.

Kodama T, Lai YY, Siegel JM (1998) Enhanced glutamate release during REM sleep in the rostromedial medulla as measured by in vivo microdialysis. Brain Res 780:178-181.

Koller EJ, Pasnau RO, Rubin RT, Naitoh P, Slater GG, Kales A (1969) Psychological, psychophysiological, and biochemical correlates of prolonged sleep deprivation. Am J Psychiatry 126:488-497.

Kwiat GC, Basbaum AI (1992) The origin of brainstem noradrenergic and serotonergic projections to the spinal cord dorsal horn in the rat. Somatosens Mot Res 9:157-173.

Lai YY, Clements JR, Wu XY, Shalita T, Wu J-P, Kuo JS, Siegel JM (1999) Brainstem projections to the ventromedial medulla in the cat: retrograde transport horseradish peroxidase and immunohistochemical studies. J Comp Neurol 408:419-436.

Leung CG, Mason P (1999) Physiological properties of raphe magnus neurons during sleep and waking. J Neurophysiol 81:584-595.

Lovick TA (1989) Systemic and regional haemodynamic responses to microinjection of 5-HT agonists in the rostral ventrolateral medulla in the rat. Neurosci Lett 107:157-161.

Magoun HW (1944) Bulbar inhibitory and facilitation of motor activity. Science 100:549-550.

Magoun HW, Rhines R (1946) An inhibitory mechanism in the bulbar reticular formation. J Neurophysiol 9:165-171.

Messier ML, Li A, Nattie EE (2002) Muscimol inhibition of the medullary raphe decreases the $\mathrm{CO} 2$ response and alters sleep in newborn piglets. Respir Physiol 133:197-214.

Messier ML, Li A, Nattie EE (2004) Inhibition of medullary raphe serotonergic neurons has age dependent effects on the $\mathrm{CO} 2$ response in newborn piglets. J Appl Physiol 96:1909-1919.

Mileykovskiy BY, Lyudmila I, Kiyashchenko I, Siegel JM (2002) Muscle tone facilitation and inhibition after Orexin-A (Hypocretin-1) microinjections into the medial medulla. J Neurophysiol 87:2480-2489.

Monti JM, Jantos H, Monti D, Alvarino F (2000) Dorsal raphe nucleus administration of 5-HT1A receptor agonist and antagonists: effect on rapid eye movement sleep in the rat. Sleep Res Online 3:29-34.

Morrison SF (2004) Activation of 5-HT1A receptors in raphe pallidus inhibits leptin-evoked increases in brown adipose tissue thermogenesis. Am J Physiol Regul Integr Comp Physiol 286:R832-R837.

Nakamura K, Matsumura K, Hubschle T, Nakamura Y, Hioki H, Fujiyama F, Boldogkoi Z, Konig M, Thiel HJ, Gerstberger R, Kobayashi S, Kaneko T (2004) Identification of sympathetic premotor neurons in medullary raphe regions mediating fever and other thermoregulatory functions. J Neurosci 24:5370-5380.

Nalivaiko E, Ootsuka Y, Blessing WW (2005) Activation of 5-HT1A receptors in the medullary raphé reduces cardiovascular changes elicited by acute psychological and inflammatory stresses in rabbits. Am J Physiol Regul Integr Comp Physiol. 289:R596-R604.

Niblock MM, Kinney HC, Luce CJ, Belliveau RA, Filiano JJ (2004) The development of the medullary serotonergic system in the piglet. Auton Neurosci 110:65-80.

Panigrahy A, Filiano JJ, Sleeper LA, Mandell F, Valdes-Dapena M, Krous HF, Rava LA, White WF, Kinney HC (1997) Decreased kainate receptor binding in the arcuate nucleus of the sudden infant death syndrome. J Neuropathol Exp Neurol 56:1253-1261.
Panigrahy A, Filiano JJ, Sleeper LA, Mandell F, Valdes-Dapena M, Krous HF, Rava LA, Foley E, White WF, Kinney HC (2000) Decreased serotonergic receptor binding in rhombic lip-derived regions of the medulla oblongata in the sudden infant death syndrome. J Neuropathol Exp Neurol 59:377-384.

Pappenheimer JR (1977) Sleep and respiration of rats during hypoxia. J Physiol (Lond) 266:191-207.

Parmeggiani PL, Rabini D (1967) Shivering and panting during sleep. Brain Res 6:789-791.

Pickel VM, Joh TH, Reis DJ (1977) A serotonergic innervation of noradrenergic neurons in nucleus locus coeruleus: demonstration by immunocytochemical localization of transmitter specific enzyme tyrosine hydroxylase. Brain Res 131:197-214.

Portas CM, Thakker M, Rainnie D, McCarley RW (1996) Microdialysis perfusion of 8-hydroxy-2-(di-n-propylamino)tetralin (8-OH-DPAT) in the dorsal raphé nucleus decreases serotonin release and increases rapid eye movement sleep in freely moving cat. J Neurosci 16:2820-2828.

Rodrigo-Angulo ML, Rodriguez-Vega E, Reinoso-Suarez F (2000) Serotonergic connections to the ventral oral pontine reticular nucleus: Implication in paradoxical sleep modulation. J Comp Neurol 418:93-105.

Sakai K, Crochet S (2000) Serotonergic dorsal raphe neurons cease firing by disfacilitation during paradoxical sleep. NeuroReport 11:3237-3241.

Simpson JM (2001) Infant stress and sleep deprivation as an aetiological basis for the sudden infant death syndrome. Early Hum Dev 61:1-43.

Skagerberg G, Bjorklund A (1985) Topographic principles in the spinal projections of serotonergic and non-serotonergic brainstem neurons in the rat. Neuroscience 15:445-480.

Sorensen E, Gronli J, Bjorvatn B, Bjorkum A, Ursin R (2001) Sleep and waking following microdialysis perfusion of the selective 5-HT1A receptor antagonist p-MPPI into the dorsal raphe nucleus in the freely moving rat. Brain Res 897:122-130.

Stornetta RL, McQuiston TJ, Guyenet PG (2004) GABAergic and glycinergic presympathetic neurons of rat medulla oblongata identified by retrograde transport of pseudorabies virus and in situ hybridization. J Comp Neurol 479:257-270.

Strecker RE, Thakkar MM, Porkka-Heiskanen T, Dauphin LJ, Bjorkum AA, McCarley RW (1999) Behavioral state-related changes of extracellular serotonin concentration in the pedunculopontine tegmental nucleus: a microdialysis study in freely moving animals. Sleep Res Online 2:21-27.

Sun C, Hildebrandt L, Curran AK, Darnall RA, Chen G, Filiano JJ (2000) Potassium permanganate can mark the site of microdialysis in brain sections. J Histotechnol 23:151-154.

Thor KB, Blitz-Siebert A, Helke CJ (1992) Autoradiographic localization of 5HT1 binding sites in the medulla oblongata of the rat. Synapse 10:185-205.

Trulson ME, Frederickson CJ (1987) A comparison of the electrophysiological and pharmacological properties of serotonin-containing neurons in the nucleus raphe dorsalis, raphe medianus and raphe pallidus recorded from mouse brain slices in vitro: role of autoreceptors. Brain Res Bull 18:179-190.

Van Bockstaele EJ, Aston-Jones G (1995) Integration in the ventral medulla and coordination of sympathetic, pain, and arousal functions. Clin Exp Hypertens 17:153-165.

Van Bockstaele EJ, Pieribone VA, Aston-Jones G (1989) Diverse afferents converge on the nucleus paragigantocellularis in the rat ventrolateral medulla: retrograde and anterograde tracing studies. J Comp Neurol 290:561-584.

Van Bockstaele EJ, Akaoka H, Aston-Jones G (1993) Brainstem afferents to the rostral (juxtafacial) nucleus paragigantocellularis: integration of exteroceptive and interoceptive sensory inputs in the ventral tegmentum. Brain Res 603:1-18.

Van der Velde L, Curran AK, Filiano JJ, Darnall RA, Bartlett D, Leiter JC (2003) Prolongation of the laryngeal chemoreflex after inhibition of the rostral ventral medulla in piglets: a role for SIDS? J Appl Physiol 94:1883-1895.

Winer BJ (1962) Statistical principles in experimental design, Ed 2, pp $309-$ 428. New York: McGraw-Hill. 